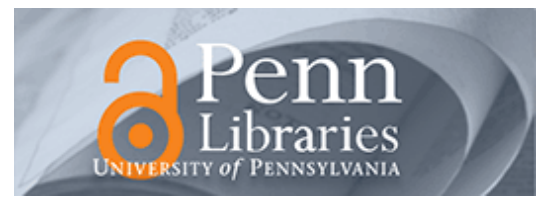

University of Pennsylvania ScholarlyCommons

\title{
Life with Ed: A Case Study of a LinuxBIOS/BProc Cluster
}

\author{
Sung-Eun Choi \\ Los Alamos National Laboratory \\ Erik A. Hendriks \\ Los Alamos National Laboratory \\ Ronald G. Minnich \\ Los Alamos National Laboratory \\ Matthew J. Sottile \\ Los Alamos National Laboratory
}

Aaron J. Marks

University of Pennsylvania, ajmarks@dsl.cis.upenn.edu

Follow this and additional works at: https://repository.upenn.edu/cis_papers

Part of the Computer Sciences Commons, and the Engineering Commons

\section{Recommended Citation}

Sung-Eun Choi, Erik A. Hendriks, Ronald G. Minnich, Matthew J. Sottile, and Aaron J. Marks, "Life with Ed: A Case Study of a LinuxBIOS/BProc Cluster", . January 2002.

Suggested Citation:

Choi, S., E. Hendriks, R. Minnich, M. Sottile and A. Marks. (2002). "Life with Ed: A Case Study of a LinuxBIOS/BProc Cluster." Proceedings of the 16th Annual International Symposium on High Performance Computing Systems and Applications. June 16-19, 2002, Moncton, NB, Canada.

This paper is posted at ScholarlyCommons. https://repository.upenn.edu/cis_papers/436

For more information, please contact repository@pobox.upenn.edu. 


\title{
Life with Ed: A Case Study of a LinuxBIOS/BProc Cluster
}

\author{
Abstract \\ In this paper, we describe experiences with our 127-no de/161-processor Alpha cluster testbed, Ed. Ed is \\ unique for two distinct reasons. First, we have replaced the standard BIOS on the cluster nodes with the \\ Lin-uxBIOS which loads Linux directly from non-volatile memory (Flash RAM). Second, the operating \\ system provides a single-system image of the entire cluster, much like a traditional supercomputer. We \\ will discuss the advantages of such a cluster, including time to boot (101 seconds for 100 nodes), \\ upgrade (same as time to boot), and start processes ( 2.4 seconds for 15,000 pro-cesses). Additionally, \\ we have discovered that certain predictions about the nature of terascale clusters, such as the need for \\ hierarchical structure,are false. Finally, we argue that to achieve true scalability, terascale clusters must be \\ built in the way of Ed.

\section{Disciplines} \\ Computer Sciences | Engineering

\section{Comments} \\ Suggested Citation: \\ Choi, S., E. Hendriks, R. Minnich, M. Sottile and A. Marks. (2002). "Life with Ed: A Case Study of a \\ LinuxBIOS/BProc Cluster." Proceedings of the 16th Annual International Symposium on High Performance \\ Computing Systems and Applications. June 16-19, 2002, Moncton, NB, Canada.
}




\title{
Life with Ed: A Case Study of a LinuxBIOS/BProc Cluster*
}

\author{
Sung-Eun Choi Erik A. Hendriks Ronald G. Minnich Matthew J. Sottile \\ Advanced Computing Laboratory \\ Los Alamos National Laboratory ${ }^{\dagger}$ \\ Los Alamos, NM 87545 \\ \{sungeun,hendriks,rminnich,matt\}@lanl.gov
}

\author{
Aaron J. Marks \\ Department of Computer and Information Science \\ University of Pennsylvania \\ Philadelphia, PA 19104 \\ ajmarks@dsl.cis.upenn.edu
}

\begin{abstract}
In this paper, we describe experienc es with our 127no de/161-processor A lpha cluster testbed, Ed. Ed is unique for two distinct reasons. First, we have replac ed the standard BIOS on the cluster no des with the LinuxBIOS which loads Linux directly from non-volatile memory (Flash RAM). Se cond, the op erating system provides a single-system image of the entir e cluster, much like a traditional supercomputer. We will discuss the advantages of such a cluster, including time to boot (101 se conds for 100 ndes), upgrade (same as time to boot), and start processes (2.4 seconds for 15,000 processes). A dditionally, we have disc over edthat certain predictions about the nature of terascale clusters, such as the ne ed for hier archial structur e, are false. Finally, we argue that to achieve true scalability, terascale clusters must be built in the way of Ed.
\end{abstract}

\section{Introduction}

Beowulf-style cluster computers gained enormous popularity because inherent in their design is the capability to lev erage bothardw are and software from

* This research was funded b y a gran $t$ from the Departmenof Energy's Office of Science and the Los Alamos Computer Science Institute. LANL LA-UR-02-1231.

$\dagger$ Los Alamos National Laboratory is operated by the University of California for the National Nuclear Security Administration of the United States Department of Energy under contract W-7405-ENG-36. the commodity computing market. Racks full of home PCs running Linux or FreeBSD (or even Windows) are a familiar sight in computer labs these days. But also inherent in their design is excess The cluster nodes have all the hardware and capabilities of being independent desktop w orkstations, when all they need is a pow er supply CPU, network interface, and the ability to spawn processes. Even specially designed cluster nodes available from major hardware vendors ship with either a floppy disk or CD-ROM drive (and sometimes ev en k eyboard, video, and mouse capabilities), the primary purpose of which is installing and upgrading system softw are.

Excess limits the ultimate scalability of a cluster in terms of system administration as well as end user performance. F or example, upgrading cluster softw are is equivalen tto upgrading software on each of the individual nodes. Starting a job to run on $P$ nodes of a cluster is equivalent to remotely logging in to eac $h$ of the $P$ nodes and starting the job. Until recently, these aspects of clustering $\mathrm{w}$ ereconsidered unimportan t. Downtime for upgrades is to be expected; job startup is not considered part of program execution time. While this may be acceptable if downtime is on the order of an hour a w eek with startup on the order of seconds, the reality is that these overheads are a function of the cluster size and grow rapidlys the number of nodes in the cluster increases. Moreover, duplicating $\mathrm{w}$ ork in troduces points of failure. If application softw are onone of the nodes is accidentally misconfigured (or configured differently from the rest), unexpected application behavior arises; if an yone of 


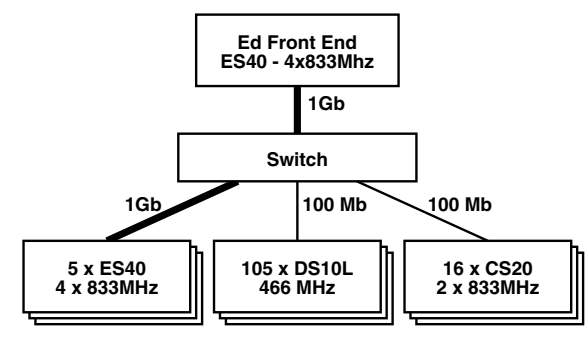

Figure 1. Ed, a Science Appliance testbed. Ed's front-end is a four processor Compaq ES40. The computer nodes are comprised of five ES40s, 105 single processor Compaq DS10s, and 16 API dual processor CS20s. The ES40s are connected to the front-end via Gigabit Ethernet, and the remainder of the nodes are connected via $100 \mathrm{Mb}$ Ethernet.

the $P$ remote $\log$ ins fails, the entire job startup fails. Current solutions to these problems all involv eadditional softw are la yers thatreate additional points of failure $[13,2,14]$.

The Cluster Research Lab at Los Alamos National Laboratory (LANL) was formed in an effort to attack these and other foreseeable problems with the next generation of cluster systems, which will beomprised of thousands of nodes and hundreds of thousands of processors. We have designed and built a prototype for a terascale-and-beyond cluster called a Science Appliance. The Science Appliance aims to enable a 1000-fold increase in the computational power of clusters with a 10 -fold decrease in the overhead of managing and using the cluster.

In this paper, w edescribe Ed, the second Science Appliance testbed. Ed is a 127-node/161-processor Alpha cluster (Figure 1). Ed's front-end is a four processor Compaq ES40. The compute nodes are comprised of five ES40s, 105 (single processor) Compaq DS10s, and 16 API dual processor CS20s. The ES40s are connect to the fron t-end via Gigabit Ethernet, and the remainder of the nodes are connected via $100 \mathrm{Mb}$ Ethernet. The compute nodes also have a single Quadrics Elan 3 interface, though none of the performance numbers reported in this paper used the Quadrics interconnect ${ }^{1}$. This mixed collection of machines and netw orks gave us a few different testbeds for our softw areall within the same cluster.

\footnotetext{
${ }^{1}$ We were unable to use the Quadrics interconnect due to bugs in the cards and vendor supplied drivers. By the printing of this paper, the Quadrics cards will have been replaced with Myrinet cards whic hwill hopefully provide a more reliable high speed interconnect.
}

Ed's compute nodes boot diskless from non-volatile memory (Flash RAM), and the operating system provides a single-system image of the entire cluster, muc $h$ like a traditional supercomputer. Some of the advantages of such a cluster include time to boot (101 seconds for 100 nodes), upgrade (same as time to boot), and start processes (2.4 seconds for 15,000 processes). F rom our experiments, w ehave also discovered that certain predictions about the nature of terascale clusters, such as the need for hierarchical structure, are false.

The remainder of the paper is organized as follows. In Section 2, we describe our prototype Science Appliance, Ed, and the technology that distinguishes it from existing clusters. In Section 3, we give preliminary performance numbers and discussion, including surprising discoveries. In Section 4, w efinish with conclusions and a discussion of future work.

\section{Building a Science Appliance}

The Science Appliance is unique in many ways. It is so different, in fact, that most of the problems of managing more traditional clusters simply donotexist. The key idea is to reduce the amount of softw are needed to run the cluster. We achieve this by lev eraging the "righ t" setof (open source) workstation soft$\mathrm{w}$ are and replacing ast amounts of redundant softw are with simpler, cluster-specific softw are. T othe maximum extent possible, we have removed scripts, configuration tools, node daemons, and the hundreds of files associated with them.

\subsection{A new cluster architecture}

The Science Appliance is a complete restructuring of the cluster architecture starting with the standard Flash RAM based BIOS responsible for individual node bring-up. Figure 2 contrasts the Science Appliance to the traditional approach to building clusters. The traditional cluster (left) is built by replicating a complete $\mathrm{w}$ orkstationinstallation on every node. The Science Appliance (right) runs an enhanced single-system image version of Lin uxcalled BProc [3]. The front-end node looks very similar to a traditional cluster node. The compute nodes run a similar kernel, but do not run an y system services and in fact do not even have direct access to program binaries. All program binaries exist only on the front-end. They are started on the front-end and moved to the nodes via a technique called directed migration (described below).

We have replaced the normal BIOS with our own BIOS, the LinuxBIOS [8]. The LinuxBIOS boots Linux 


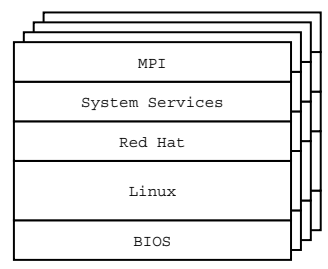

$T$ raditional cluster ardhitecture

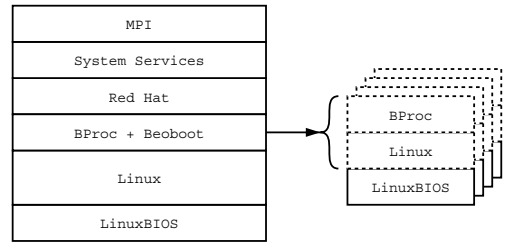

Science Appliance architecture

Figure 2. The traditional cluster architecture versus the Science Appliance. The traditional cluster (left) is built by replicating a complete workstation environment on every node. The Science Appliance (right) runs an enhanced single-system image version of Linux called BProc. The front-end node looks very similar to a traditional cluster node; the compute nodes run a similar kernel, but do not run any system services and in fact do not even have direct access to program binaries.

from the Flash memory, and starts Linux with an initial RAM disk, also loaded from Flash memory. Because we control the BIOS, we are able to control the nodes from the first instruction after reset, so the nodes function as purpose-built cluster nodes from pow er-on. Moreover, the nodes are more reliable as they only require a working pow er supplyand CPU to boot. With a working net $\mathrm{w}$ orkinterface, they are also net $\mathrm{w}$ orkmanageable from pow er-on.

In most cases, the Flash kernel is not used as the operational kernel, though it can be. The kernel loaded from Flash is typically used to contact a front-end node and prepare the machine to run the operational kernel, which it receiv es fromthe fron t-end. In other words, Lin ux is being used as a netw ork bootstrap to the frontend node running BProc.

This tw o-phased boot process, called two-kernel monte, is illustrated in Figure 3. Each node begins by requesting an IP address, boot image (kernel) filename, and port numbers (steps 1 and 2). The nodes then request the operational kernel, which is distributed using IP multicast (steps 3 and 4). Note that a single multicast message (dashed line) could be used by all the nodes. Since the nodes cannot be expected to simultaneously reach this step, they begin receiving multicast pac ketsimmediately, even if the transmission of the message has already begun. Nodes that begin receiving part-way through the boot image continue into the next multicast message until they have a complete image. Though this implies that more than one multicast message may be sent, in practice the number of copies transmitted is far less than the number of noew. Finally, the nodes boot the operational krnel and again request an IP address (steps 5 and 6 ), and establish a TCP connection to the front-end (not shown).

Notice that there is an explicit handshake betw een each node and the front-end betw een steps 1 and 2 and again between steps 5 and 6 . It was our anticipation that these handshakes $\mathrm{w}$ ould require us to add hierarc hy tohe managemen $\mathrm{t}$ structure (i.e., multiple front-end nodes) for clusters larger than 100 nodes. In fact, hierarchical cluster structure has been the rule for many existing production-grade cluster systems $[11,1]$. In the next section, we show why we no longer believe that hierarchical structure is necessary for cluster with $\mathrm{h}$ undreds of nodes.

Here we see an immediate advan tage of using Linux as the netw ork bootstrap medanism: w e can exploit a protocol (IP Multicast) that reduces the netw ork o verhead for booting from $O$ (\# nodes) to $O(1)$. Contrast this with the widely used approach of using PXE [4] for net work boot. PXE is very limited as to which interfaces (a limited number of Ethernet interfaces), protocols (one), and authentication mechanisms (none) it supports, where as the Science Appliance can support any thing Linux can support because Linux is our bootstrap mechanism. The PXE boot protocol is both naive and insecure, whereas the Science Appliance protocol can be as secure as the most secure netw ork protocols Lin ux can support.

The root file system is maintained in a RAM disk. Nodes actually come up with an empty file system. The few files required for normal operation (a few entries in /etc, /dev) are created or downloaded from the fron $t$ end at boot time. Dynamically linked libraries and the dynamic linker itself are downloaded on demand from the front end via multicast. Consequently, there are no issues with version skew of binaries or libraries.

Contrast this with the tw o most popular approades of maintaining the root file system: local disk or NFS. The local disk is very susceptible to version skew because each local disk must be individually installed and 


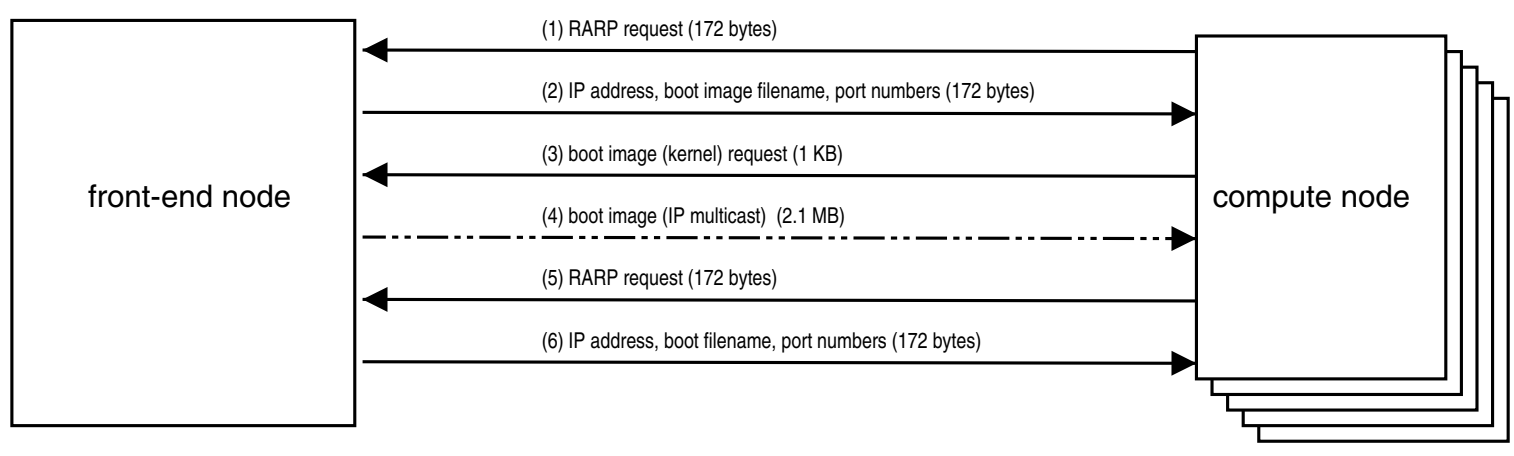

Figure 3. Cluster node boot process. Each node begins by requesting an IP address, boot image (kernel) filename, and port numbers (steps 1 and 2). The nodes then request the operational kernel, which is distributed using IP multicast (steps 3 and 4). Note that a single multicast message (dashed line) could be used by all the nodes. Finally, the nodes boot the operational kernel and again request and IP address, boot image (kernel) filename, and port numbers (steps 5 and 6).

configured in exactly the same manner. Keeping all the local disks synchronized requires a complex tool chain and continuous manual management of configuration files. In a Science Appliance, if there is a working local disk, it can be used for data files, but it is not necessary for node operation. We have found that one of the most common failures on our clusters is the local disk. Since a Science Appliance node will boot even if the local disk fails, it can notify the front-end of the failure and proper action can be taken.

Though they eliminate the version skew problem, it is fairly common kno wledgethat NFS-based root file systems are not a scalable technology beyond 100 nodes. F or large scale clusters, this scaling limitation requires that the cluster be arranged in sub-clusters of 32 or 64 nodes, with each domain served by a local NFS server. In the case of a 1024-node Tru64 Alpha cluster maintained by another group at LANL, there are 32 separate NFS domains, each requiring a primary and bac kupserver. Sandia National Laboratories demonstrated that scaling NFS for CPlant [11], one of the largest production clusters in existence, required the creation of whole new NFS subsystems.

\subsection{Other technologies}

On top of the basic architecture for the Science Appliance w ehave added other technologies that contribute to a final cluster environment. Here we briefly describe two: Supermon, a cluster monitoring tool, and edb, a scalable parallel debugger.

Supermon Supermon is a high speed cluster monitoring tool, able to achiev e samplingrates never seen before $[9,12]$. The dramatically lower ov erheard coupled with the higher precision of Supermon as compared to the standard Linux monitoring tools such as rstatd allows us to see behavior that was previously invisible. Supermon consists of tw o data collection programs, a loadable kernel module, and a library for managing the data. The first program, "mon," is a data server that runs on each node and does primary data collection. Mon can accept many connections to remote data gathering programs. Mon also supports dynamic filters so that only the data needed by remote programs need move over the network. The second program, "supermon," is a data concentrator that can collate the data from many mons so that user programs do not need to manage this task. F urthermore, since the protocol used by mon and supermon is compatible, supermon programs can be composed into a hierarc hy to optimize communications based on the cluster interconnect topology (though we have not seen the need for this on Ed). The kernel module provides additional entries in /proc which are read by mon. Finally, the libraries support a data manipulation API so that users can easily dev elop programs for analysing data. We have data filters for Perl, Ja wa, MatLab, and other programs. Some programs provide a GUI-style interface for interactiv e real-time monitoring, and other programs are used for off-line data analysis of supermon tracefiles.

Edb Edb is an interactiv eparallel debugger for at scale application debugging [5]. Edb is currently implemented usinghe UNIX ptrace() system call. On most clusters using ptrace w ould limit edb to run- 
ning on a single node. Since BProc transparently supports ptrace operations over the netw orkto remote nodes, edb can control processes on all the nodes in Ed. It uses the bfd library to acquire the target symbol table, the proc filesystem to query the status of running processes and the readline library for an enhanced, Emacs-like command-line interface (which includes command history and completion). Debugging commands exist for loading and running a parallel application, process control (e.g., wait, continue, signals), examining and modifying state and process selection. All debugging commands are executed on the current process group (called a process focus). Users can create, name, and use as many process foci as needed. By default, the process focus is all processes, but this can be modified at any time during the debugging session.

\subsection{Summary}

The Science Appliance effectively reuses commodity desktop hardware and softw are. The design eliminates vestigial elements of PC clusters such as the BIOS and local or NFS-mounted root, while lev eraging existing softw are suc $h$ as the Linx operating system. Removing dependence on the BIOS and local or NFS-mounted root makes for enormous improvements in reliabilit y and greatly reduces the management effort, as well as eliminating all the scripts, tools, and documentation needed for keeping nodes in sync. Leveraging Lin ux enables the use of the most appropriate and best available netw ork driers, protocols, as well as programming tools.

\section{Performance Evaluation}

In this section, we present a performance evaluation of our Science Appliance, Ed, with respect to installation and upgrade time, job startup time, Supermon, and edb.

\subsection{Installation and upgrade (boot) time}

Ultimately, it is our hope that all vendors will sell cluster nodes with LinuxBIOS pre-installed. Currently tw osuch vendors exist (Lin uxLabs and Linux Netw orx), and only one (Linx Labs) offers BProc extensions to the installed operating system. For Ed, LinuxBIOS had to be installed manually which involv ed setting up a standard network boot on each node. Once the nodes came up as part of the cluster, the Flash RAM was ov erwritten with LinuxBIOS from the frontend node over the netw ork. This was the first and only

\begin{tabular}{|r||r|r|}
\hline nodes & bo ot time & time per additional node \\
\hline 1 & 69 seconds & N/A \\
\hline 10 & 71 seconds & 0.22 seconds \\
\hline 100 & 101 seconds & 0.32 seconds \\
\hline
\end{tabular}

Table 1. Boot time from power-on to node ready for one, ten, and 100 DS10 nodes and the time to boot each additional node.

time we had to write the Flash because the operational kernel is downloaded from the front-end node.

Once a node has been booted by LinuxBIOS, it contacts the front-end node for the operational kernel and reboots itself. Upgrading the cluster nodes is achiev ed by building a new operational kernel on the front-end and rebooting the cluster nodes.

In the previous section, w ementioned the current belief that hierarchical structure is necessary for clusters larger than 100 nodes. We found that using multicast capabilities changed our view on hierarc hical structure.

Table 1 shows the time to boot one, ten, and 100 DS10 nodes and the time to boot each additional node. Using a faster netw ork, w ebelieve these boot times will improve to better than 8 minutes (based on extrpolation from the current numbers). As a reference point, prior to adding multicast functionality, we were unable to boot 100 nodes simultaneously due to time outs induced by excessive load on the network and the front end machine. Another 128-node Alpha cluster at LANL running Tru64 cannot be booted in less than two hours.

\subsection{Job startup time}

Job startup time is crucial to ov erall application performance. Consider the case of a 10-hour sequential job running on a cluster with 100 nodes. T oachieve the full capability of the cluster, scalar ov erheads should be less than 1/100 the ideal parallel runtime, i.e., perfect speed-up. Since the ideal parallel runtime is 6 minutes, the limit on scalar overhead is 3.6 seconds. Y et te have clocked MPI startup times on a cluster this size at 15 seconds. The problem only gets worse as we grow the cluster to 1024 nodes, which would in turn limit scalar overhead to less than $1 / 2$ second.

Table 2 compares remote job fork using a flat (sequential) or tree-based startup for up to 15,000 processes on 120 nodes. For small numbers of small processes, a linear start-up is faster. This is partly due to the fact that the front end machine is connected via 


\begin{tabular}{|r||c|r|r|}
\hline $\begin{array}{c}\text { job size } \\
\text { (processes) }\end{array}$ & $\begin{array}{c}\text { process } \\
\text { size }\end{array}$ & $\begin{array}{c}\text { linear } \\
\text { spawn }\end{array}$ & $\begin{array}{c}\text { tree } \\
\text { spawn }\end{array}$ \\
\hline 10 & $150 \mathrm{k}$ & 0.03 & 0.06 \\
\hline 100 & $150 \mathrm{k}$ & 0.36 & 0.18 \\
\hline 1000 & $150 \mathrm{k}$ & 5.00 & 0.24 \\
\hline 10000 & $150 \mathrm{k}$ & 63.00 & 1.54 \\
\hline 15000 & $150 \mathrm{k}$ & 81.00 & 2.40 \\
\hline \hline 10 & $5 \mathrm{MB}$ & 0.81 & 1.5 \\
\hline 100 & $5 \mathrm{MB}$ & 7.40 & 4.8 \\
\hline 1000 & $5 \mathrm{MB}$ & 90.00 & 4.8 \\
\hline 10000 & $5 \mathrm{MB}$ & 837.00 & 4.9 \\
\hline
\end{tabular}

Table 2. Job startup times in seconds. Linear spawn starts every process on the front end and migrates it to a node. Tree spawn replicates processes in a tree structure to exploit parallelism in the network.

Gigabit Ethernet and the nodes are connected via fast Ethernet. The tree spawn's time becomes basically flat once the number of processes reac hes the $n$ umber of nodes in the system. This is because the tree spawner uses local fork once it gets a single process on a node. Each one of those forks creates a new child which must be represented on the fron tend machine so there is communication for each one of those forks. Due to the apparent lack of overhead there, w ebeliev ethe tree based job startup will scale very w ell to large (thousands) numbers of machines.

Notice that while job startup is hierarchic al, the cluster structure itself do es not ned to be hierarchical.

In previously published work, it was sho wn that $\mathrm{Su}-$ permon can collect 100 samples per second without noticeably affecting node performance [9]. More recen tly, improvements to the data protocol and node data source drastically increased this efficiency [12]. Supermon is composed of three levels through which data is filtered and passed back to clients. The low est level, a kernel module providing additional en tries in /proc, provides the raw monitoring data at a maximum sampling rate of $3500 \mathrm{~Hz}$ on Ed's nodes (up to $6600 \mathrm{~Hz}$ on Intel platforms). The single node TCP data server (mon) takes this data, filters it, and passes it to clien ts at a maximum sampling rate of $1400 \mathrm{~Hz}$. Finally, a data concentrator (supermon) collects data from mon servers and returns it to clients over TCP at a maximum sampling rate of $750 \mathrm{~Hz}$ for a single node. These rates, sho wnin Figure 4, reflect the entire 1K per node data set being transferred, and are higher for filtered subsets of this data.

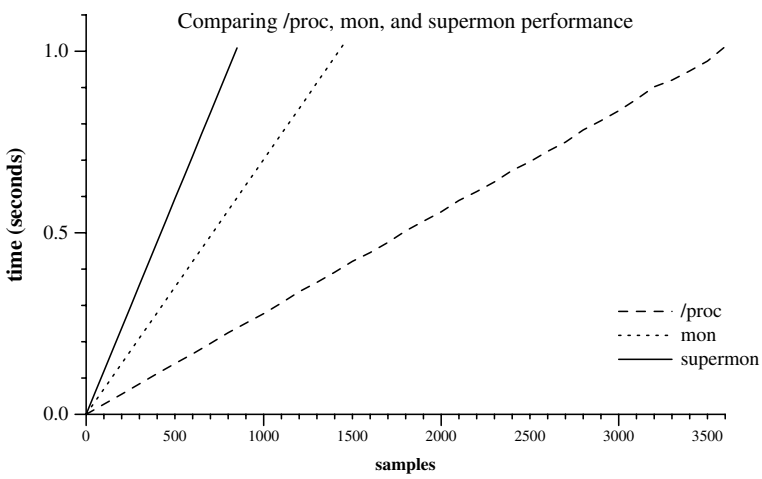

Figure 4. Comparison of /proc, mon, and supermon sampling rates for single node monitoring

\subsection{Edb scalability}

T o demonstrate the scalability of edb, we measured its response time for startup and continuation of 15,000 processes. Startup includes distribution and process initialization of the target application and contin uation includes the issue and return of a ptrace continue command for all processes. F or an interactive debugging session with 15,000 processes, we measured 40 seconds for startup and 10 seconds for continuation.

\section{Conclusions and F utureWork}

The current state-of-the-art technology for building cluster computers will not be usable for terascale-andbeyond systems due to the direct relationship betw een cluster size and cluster management and programming overhead. In this paper, w eha vepresented our experience with a protot ypeScience Appliance, Ed, a testbed for future generation cluster computers. We have sho wn that Lin uxBIOS, a replacement for the standard PC BIOS, can be used as a netw ork boostrap to bring up the cluster nodes. The front-end node provides a single-system image of the cluster, much like a traditional supercomputer, and thus the nodes can be conveniently and efficiently managed from the frontend. Our Science Appliance can be booted in under two minutes, which means that it can be upgraded in under two minutes. The front-end can support 15,000 processes which can be started on the computed nodes in 2.4 seconds, using a simple tree-based startup. Finally, we have built t w o clusterva are tools, Supermon and edb, which also scale well to large clusters.

Our initial experiences with Ed have shown us that hierarchical structure, already being used in produc- 
tion clusters today, may not be necessary for clusters up to 1000 nodes if they are built in the way of Ed. Notice that hierarc hy is neededfor job startup, but this does not require hierarchical structure.

Compared to LANL's other experiences with vendor supported clusters of similar size and make, Ed is several orders of magnitude easier to manage and maintain. Consequently, we can only advocate building terascale-and-beyond clusters as Science Appliances.

\subsection{Future Work}

By the prin ting of this paper, Ed's high speed interconnect will have been replaced with Myrinet. We expect to see even more impressive numbers for boot time and start up using Myrinet (versus 100 MB Ethernet). Our next steps with this cluster will include additional scaling w ork, a scheduler, and file system research.

Lighter weight boot process The current boot process involvesrunning scripts on the fron t-end to setup the nodes. These scripts are currently complex and involve a fair amount of processing per node on the fron t-end. Replacing these scripts with equivalen t programs which can do the same work on the slave node instead of the front-end would considerably lighten the fron t-end's load at boot time.

BProc Job Scheduler BProc's single-system image requires a new job scheduler. The interesting aspect of the sc heduler is that the nodes hav e attributes similar to files and thus access to the nodes can be trivially managed from the fron tend. Unlike existing cluster schedulers, there is no need to contact the individual nodes. In additionfast boot times open up other interesting possibilities such as rebooting the nodes after each job for securit y reasons or booting different kernels for different jobs.

Private Name Spaces Current research in cluster file systems attempt to provide a global file system for the entire cluster. Our belief is that this is inherently unscalable. We are currently implementing Plan 9 [10] style Priv ateName Spaces for Lin ux[6, 7]. We will incorporate this into the next Science Appliance environment.

\section{References}

[1] Argonne National Laboratory. City - The MCS Large Cluster System Software Toolkit.
[2] G. Bruno and P. M. P apadopoulos. NPA CIRocks: T ools and Techniques for Easily Deploying Manageable Linux Clusters. In Proceedings of Cluster 2001, Anaheim, CA, October 2001.

[3] E. A. Hendriks. The Beowulf distributed process space. Submitted for public ation 2002.

[4] Intel Corporation. Preboot execution environment (PXE) specification.

[5] A. J. Marks. T ow ard the design and implemetration of a scalable debugger. (In preparation), 2001.

[6] R. Minnich. 9.2.u: A user-mode private name space system for unix. Technical report, Defense Advanced Research Projects Agency, September 1998.

[7] R. Minnich. Private namespaces for linux. Dr. Dobb's Journal, December 2001.

[8] R. Minnich, J. Hendricks, and D. Webster. The Linux BIOS. In Proceedings of the Fourth Annual Linux Showcase and Conference, Atlan ta, GA, October 2000.

[9] R. Minnich and K. Reid. Supermon: High performance monitoring for linux clusters. In Proceedings of the Fifh A nnualLinux Showcase and Conference, Oakland, CA, November 2001.

[10] R. Pike, D. Presotto, S. Dorward, B. Flandrena, K. Thompson, H. Trickey, and P. Winterbottom. Plan 9 from Bell Labs. Computing Systems, 8(3):221-254, 1995 .

[11] R. Riesen, R. Brightwell, L. A. Fisk, T. Hudson, J. Otto, and A. B. Maccabe. Cplant. In Proceedings of the Second Extreme Linux Workshop, June 1999.

[12] M. J. Sottile and R. G. Minnich. Supermon: Cluster monitoring as if performance mattered. Submitted for public ation 2002.

[13] The Open Cluster Group. OSCAR: A packaged cluster softw are stack for high performance computing.January 2001.

[14] T urboLinx. Turbolinux P overCockpit T ednology Overview (white paper). September 2001. 